\title{
CryoEM Structural Analysis of Defensin Neutralization of Adenovirus
}

\author{
Robert J. Kim, ${ }^{*}$ Justin W. Flatt,* Steffen Lindert, ${ }^{*}$ Dewight R. Williams, ${ }^{*}$ Jason G. Smith, ${ }^{* *}$ Glen \\ R. Nemerow, *** and Phoebe L. Stewart* \\ * Department of Molecular Physiology and Biophysics, Vanderbilt University Medical Center, \\ Nashville, TN 37232 \\ ** Department of Microbiology, University of Washington School of Medicine, Seattle, WA 98195 \\ *** Department of Immunology and Microbial Science, The Scripps Research Institute, La Jolla, \\ CA 92037
}

Human adenoviruses (HAdVs) are a major cause of acute respiratory, intestinal and ocular infections. These viruses are often chosen as candidate gene therapy vectors because of their ability to infect a wide range of tissues and the fact that they can be produced in high titers in the laboratory. There are over 50 known types of HAdV. Understanding the molecular mechanisms underlying the host immune response to adenovirus is of importance for designing novel, welltargeted vectors. Our study focuses on the mechanism by which adenovirus is neutralized by human alpha defensin 5 (HD5), a small peptide of the innate immune system.

Defensins are small, positively charged peptides that bind to and inactivate bacterial and viral pathogens through various membrane and capsid interactions. HD5 neutralizes HAdV by preventing virus uncoating and release of the membrane lytic protein VI during cell entry [1]. A recent study has shown that defensin molecules bind with low micromolar affinity to sensitive HAdV types [2]. Sensitivity to HD5 neutralization was found for many types from HAdV species A, B, C, and E, while all of the types that were tested from HAdV species D and F were resistant. A $12 \AA$ resolution cryoEM structure of the Ad5.F35 vector in complex with HD5 (produced by mixing the virus with $20 \mu \mathrm{M}$ HD5) showed numerous binding sites over the HAdV capsid [2]. The cryoEM structure in combination with a sequence analysis of capsid proteins from sensitive and resistant HAdV types led to a hypothesis that the critical HD5 binding site is located at the region between the fiber and penton base proteins. This was supported by infectivity studies with virus chimeras comprised of capsid proteins from sensitive and resistant HAdV types.

Here we present a $9.7 \AA$ resolution cryoEM structure of the Ad5.F35/HD5 complex based on a dataset with 3,515 particle images collected on an FEI Polara (300kV, FEG) cryoelectron microscope. For this study we used a more physiologically relevant 5uM concentration of HD5, eliminating most of the nonspecific binding of HD5 on the HAdV capsid. Comparison of the Ad5.F35/HD5 structure with a control structure of Ad5.F35 [3] reveals density between the Ad5.F35 fiber and penton base (Fig. 1). Modeling with the crystallographic structure of HD5 and structures of the HAdV capsid proteins indicates that HD5 is likely bound to partially flexible sites on the fiber and penton base (Fig. 2). Our immediate goal is to build a model for HD5 bound to the fiber/penton base complex that can serve as input to MDFF, a molecular dynamics program designed for flexible fitting of coordinates within cryoEM density [4]. Our long term goal is to understand the molecular interaction between HD5 and sensitive HAdV types [5].

References

[1] J.G. Smith and G.R. Nemerow, Cell Host Microbe 3 (2008) 11. 
[2] J.G. Smith et al., PLoS Pathog. 6 (2010) e1000959.

[3] S.D. Saban et al., J. Virol. 80 (2006) 12049.

[4] L.G. Trabuco et al., Methods 49 (2009) 174.

[5] This research was supported by an NIH grant to PLS (R01 AI042929).
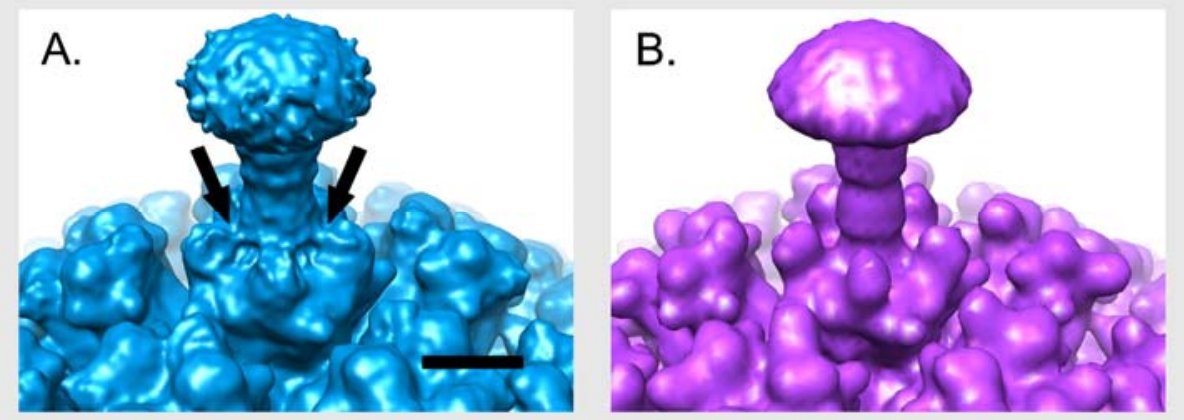

FIG. 1. Comparison of vertex regions from Ad5.F35 cryoEM structures with and without HD5. (A) Ad5.F35 in the presence of $5 \mu \mathrm{m}$ HD5. Arrows indicate areas of extra density between the fiber shaft and penton base not observed in the control Ad5.F35 structure. (B) Ad5.F35 in the absence of HD5 [3]. Scale bar represents $50 \AA$.

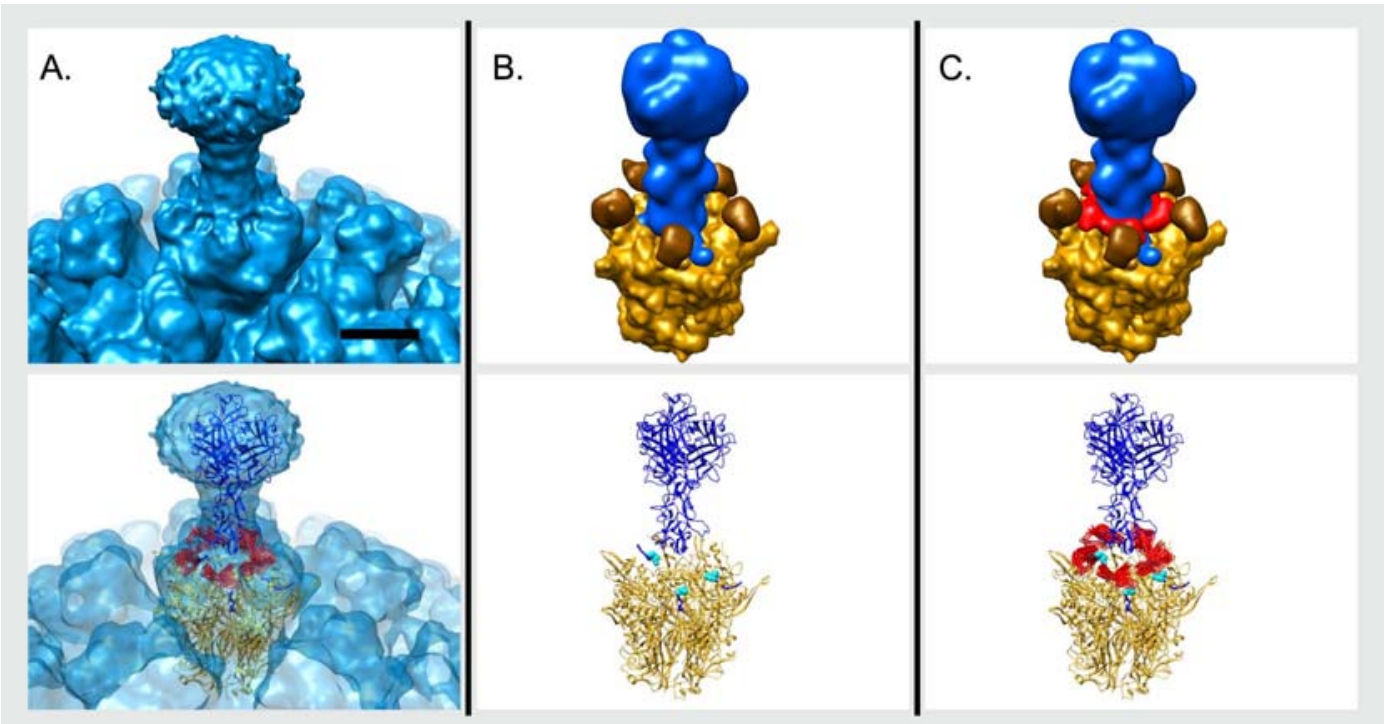

FIG. 2. Modeling of the HAdV vertex with and without HD5. (A) Vertex region of the cryoEM structure of Ad5.F35/HD5 shown with a solid (top) or transparent (bottom) isosurface. Crystal structures of penton base (gold), fiber (blue), and HD5 (red) are overlaid in the bottom panel. (B) Simulated density (top) for the Ad5 penton base (gold) and its flexible RGD loops (brown), together with the Ad35 fiber (blue) of the Ad5.F35 pseudotyped vector. The corresponding atomic models are shown in the bottom panel. Two of the four fiber residues (ED of the EDES sequence) predicted to be involved in HD5 binding [2] are shown in cyan. (C) The same as in panel B with simulated density and coordinates for HD5 (red). Multiple overlapping positions are shown for HD5 to simulate the observed cryoEM density. PDB IDs of the structures shown are 1X9T for penton base and 1ZMP for HD5. The Ad35 fiber is modeled from 1X9T and 1QIU. Rosetta was used to build models for the flexible RGD loops of penton base. Scale bar represents $50 \AA$. 